\title{
Interval-Based Representation of Spatio-Temporal Concepts ${ }^{\star}$
}

\author{
Toshimi Tagashira Toshiyuki Amagasa \\ Masayoshi Aritsugi Yoshinari Kanamori \\ Department of Computer Science, Gunma University \\ 1-5-1 Tenjin-cho, Kiryu 376 Japan \\ \{tagasira,amagasa,aritsugi,kanamori\}@dbms.cs.gunma-u.ac.jp
}

\begin{abstract}
This paper proposes a. method for representing numerous spatio-temporal concepts of objects appearing in image sequences. The objects are modeled approximately, by use of cuboids consisting of minimum bounding rectangles with time intervals. The spatial relations, including topological and directional relations and the distances between objects, are then defined. The definitions allow for uniform representations of the spatio-temporal queries on objects appearing in image sequences using intervals. This paper also gives examples of such queries for contents retrieval, and illustrates how they are performed in our method.
\end{abstract}

\section{Introduction}

In the retrieval of images containing objects, it is essential to model the objects appropriately. There are many researches employing two-dimensional minimum bounding rectangles (MBRs) to model objects for the content descriptions of images $[4,11,12,13,14]$. While obviously not capable of describing an object's features in precise detail, MBRs can provide a simple description of the size and position of an object. In this paper, we also employ the MBR approach to model objects appearing in an image, and exploit minimum bounding cuboids (MBCs), which consist of MBRs with time intervals, during which the objects are supposed to exist as they are, to represent three-dimensional spatio-temporal concepts.

An MBR consists of two intervals on the axes of coordinates. Thus, the relations between MBRs can be represented by combining the two relations between intervals. This paper employs MBCs to model objects appearing in image sequences, and proposes a method of representing spatio-temporal relations between MBCs. Further, we here provide definitions of numerous spatial relations, including topological and spatial relations and the distances between MBCs. Based on the definitions we can express spatio-temporal queries on objects appearing in image sequences, and can derive the answers for the queries from MBC data.

The definitions of the spatial relations are based on the relations between two temporal intervals listed by Allen [1]. We extend Allen's relations so that a relation can have exact degree concerning two intervals. Because the degree can

\footnotetext{
* This work was supported in part by the Ministry of Education, Science, Sports and Culture in Japan under a Grant-in-Aid for Scientific Research on Priority Areas (Grant-No. 08244101), and in part by Sun Microsystems, Inc.
} 
be calculated directly from the intervals, our extension requires no additional data storage. The exact degree of distance between objects can be derived by the extension, as will be shown.

Just as MBRs have frequently been used to model objects, so too have Allen's relations been used to represent the spatial relations between them $[5,12]$. We do not claim these as contributions of this paper. Rather, the main contributions are:

- The use of intervals to uniformly express spatio-temporal queries. Answers to queries are derived from interval data stored in a database. Because intervals have already been well-studied by numerous researchers $[5,12]$, they can be employed fairly easily.

- The first expression of several directional relations between objects. These relations have not previously been accounted for in other research.

The definitions we give in this paper allow for the expression and processing of spatio-temporal queries based on intervals. Previously proposed spatial queries include Spatial SQL [6], a language-based query that is useful, for example, in finding a closest object. Numerous proposals have also been made for expressing temporal queries. In TSQL2 [15], for example, temporal queries might be used to determine all employees at work over a given time interval. These studies of spatial and temporal expression have presented a number of sophisticated models and languages. Nonetheless, further investigation is needed into the expression of spatio-temporal relations and queries. Needless to say, the improved synthesis of the spatial and temporal expressions of objects will be critical for better temporal geographic information systems [3], video data [4, 8], and related technologies.

There are several directional relations between objects that have not been taken into account so far. For example, let us assume that there are two objects and the topological relation between them is contains, as given by Allen. In this case, the directional relation between the objects cannot be expressed using the relations in problematic images. Accordingly, we aim to extend Allen's original series, making it possible to express such problematic spatial relationships. Moreover, the extension enables us to express the exact degree of dynamic changes appearing in image sequences.

The remainder of this paper is organized as follows. Section 2 proposes an approach for modeling image sequences. Section 3 shows spatio-temporal relations between objects that appear in image sequences. Section 4 gives an example of images and their corresponding queries. Section 5 compares related work with ours, and Section 6 concludes this paper.

\section{Image Sequences}

An image sequence is a collection of images with individual creation times by which they can be ordered. Image sequences might include, for example, frames of video data, or a series of X-ray photographs collectively forming a case history.

In this paper, we focus on spatial relations between two objects that appear in an image, and on changes of the relations over time. Objects appearing in images are supposed to be detected and modeled properly with MBRs: this study does not mention concerning them.

\subsection{Time Intervals}

First, we give a brief explanation of the time-interval based conceptual model $[7,2]$ that we exploit to express temporal relations in this study. The time-interval 
based conceptual model allows us to represent the history of a real-world event, such as a medical treatment history, as a collection of time intervals. In such a history, we may have to consider not only time intervals in which a particular medication or treatment is used but also those in which they are not used. To this end, the data model introduces real-time intervals and null-time intervals, which are defined as follows.

\section{Definition 1 Real-time intervals and null-time intervals}

Let $e_{i}(1 \leq i \leq n)$ be an event, that is, $e_{i}$ denotes that the event is going on. In contrast, $\overline{e_{i}}$ denotes that the event is suspended. Let $E \subseteq \mathcal{E}=\left\{e_{1}, e_{2}, \ldots, e_{n}\right\}$ be a set of events. $\bar{E}$ represents $\left\{\overline{e_{i}} \mid e_{i} \in E\right\}$.

Let $t_{s}$ and $t_{e}\left(t_{s} \leq t_{e}\right)$ be time instants. A real time interval is defined as $t=\left(t_{s}, t_{e} ; E\right)$, and a null time interval is defined as $t=\left(t_{s}, t_{e} ; \bar{E}\right)$.

Allen listed the thirteen relations between two time intervals [1]. This study treats time as a discrete value: that is, a time corresponds to a natural number. The time-axis is given by a list of non-decomposable units that are called chronons [9]. Thus, for instance, the fact that the relation between two time intervals, $t=\left(t . s t p, t . e t p ; E_{t}\right)$ and $u=\left(u . s t p, u . e t p ; E_{u}\right)$, is meets means the following: $\quad t$ meets $u$ iff t.etp $+1=u . s t p$.

Let us next consider a collection of time intervals.

\section{Definition 2 Composite-time intervals}

Let $_{i}$ (i is odd, $1 \leq i \leq n$ ) be a real time interval, and $t_{i}$ (i is even, $2 \leq i \leq n$ ) be a null time interval.

$A$ composite time interval $C$ is defined as

$$
C=\left(\ldots\left(\left(t_{1} \text { meets } t_{2}\right) \text { meets } t_{3}\right) \ldots \text { meets } t_{n}\right)
$$

Note that not only temporal queries but also complex temporal constraints can be expressed by applying operations to collections of time intervals. Such expression, however, is beyond the scope of this paper. Please refer to $[7,2]$ for more details.

\subsection{MBRs and MBCs}

We model an object appearing in a 2-D image using the two intervals that are made of projections of the object on the axes of coordinates (Fig. 1) ${ }^{2}$.

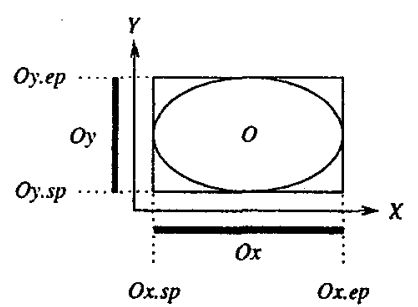

Fig. 1. A minimum bounding rectangle (MBR)

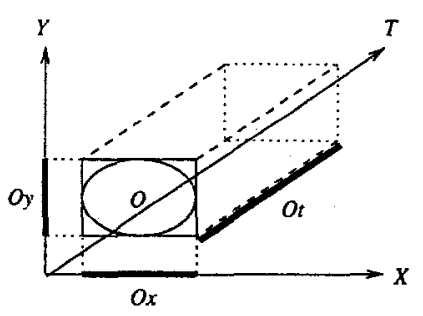

Fig. 2. A spatio-temporal cuboid, or a minimum bounding cuboid (MBC)

Just as with time values, we model a value on each axis as a discrete value. Each axis is a list of non-decomposable units that are called quantum [9]. Any value on either axis can be expressed with quantum. An interval is defined as

${ }^{2}$ Clearly, the MBR modeling approach can only approximate the forms of objects. This drawback is not addressed in this paper, but can be pursued in $[14,13]$. 
a closed one and also as a collection of contiguous quanta. The duration of an interval is used to represent its size: that is, the number of quanta included in the interval.

\section{Definition 3 Intervals}

Let $i_{s}$ and $i_{e}$ be points on an axis of coordinates. Let us define an interval as a closed interval $\left(i_{s}, i_{e}\right)$.

Definition 4 Duration

Let $i=\left(i_{s}, i_{e}\right)$ be an interval. The duration of the interval $|i|$ is defined as

$$
|i|= \begin{cases}i_{e}-i_{s}+1 & \text { if } i_{s} \leq i_{e} \\ 0 & \text { if } i_{s}>i_{e}\end{cases}
$$

In this paper, we refer to an interval $i$ of which duration is 0 as $\varepsilon$.

Definition 5 Minimum bounding rectangles (MBRs)

Given object $O$, let $O x=(O x . s p, O x . e p)$ and $O y=(O y . s p, O y . e p)$ be its intervals, created by projecting it on $X$ and $Y$ axes, respectively. The minimum bounding rectangle of object $O$ is defined as the domain $(x, y)$ where

$$
\text { Ox.sp } \leq x \leq \text { Ox.ep } \wedge \text { Oy.sp } \leq y \leq \text { Oy.ep . }
$$

This is described as $(O x, O y ; O)$ in this paper.

We consider that minimum bounding rectangles appearing on an image continue to exist from the creation time of that image to the generation time of the next (Fig. 2).

To take the time interval into consideration, we define spatio-temporal cuboids, or minimum bounding cuboids (MBCs), as follows.

\section{Definition 6 Spatio-temporal cuboids, or minimum bounding cuboids (MBCs)}

Given object $O$, the minimum bounding rectangle of which is $(O x, O y ; O)$. Let the time interval in which $(O x, O y ; O)$ is available be $O t=($ Ot.stp, Ot.etp $)$. Let us define that the object's spatio-temporal cuboid is the domain $(x, y, t)$ where

$$
\text { Ox.sp } \leq x \leq O x . e p \wedge O y . s p \leq y \leq O y . e p \wedge \text { Ot.stp } \leq t \leq \text { Ot.etp } .
$$

This is described as $(($ Ot.stp, Ot.etp); $((O x . s p, O x . e p),(O y . s p$, Oy.ep $) ; O))$, $(O t ;(O x, O y ; O))$, or $(O x, O y, O t ; O)$ in this paper.

Note that an MBC $(\varepsilon, \varepsilon, O t ; O)$, or $(\varepsilon, \varepsilon,($ Ot.stp, Ot.etp, $\bar{O}) ; O)$, is used to represent that object $O$ does not exist in interval $O t$.

To model changes of objects appearing in an image sequence, including the objects' movement, expansion, contraction, appearance and disappearance, we introduce composite spatio-temporal cuboids (Fig. 3).

\section{Definition 7 Composite spatio-temporal cuboids}

Given an image sequence consisting of $n$ images, each numbered as $i(1 \leq i \leq$ $n)$. Given object $O$, the spatio-temporal cuboid Cu $u_{i}$ of which is $\left(O x_{i}, O y_{i}, O t_{i} ; O\right)$. The object's composite spatio-temporal cuboid is defined as

$$
\left\{C u_{1}, C u_{2}, \ldots, C u_{n} \mid\left(\ldots\left(\left(O t_{1} \text { meets } O t_{2}\right) \text { meets } O t_{3}\right) \ldots \text { meets } O t_{n}\right)\right\} \text {. }
$$




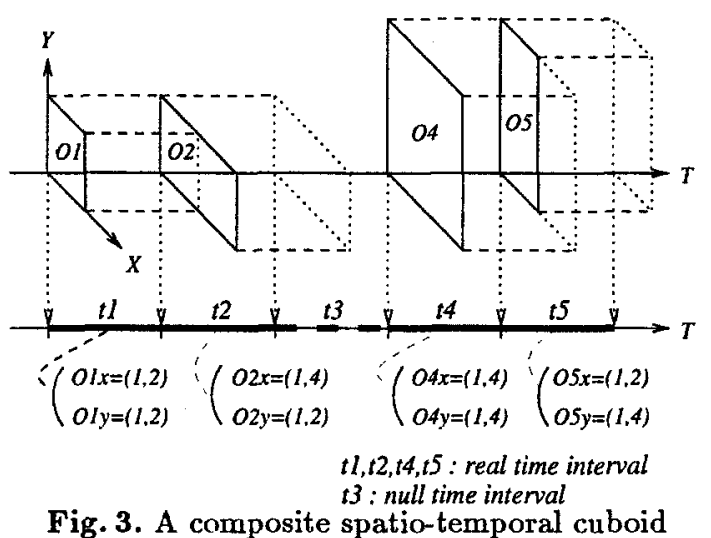

\section{Spatio-Temporal Relations between Objects}

\subsection{Spatio-Temporal Cuboid Relations}

We extend Allen's relations between intervals [1] in order to express relations between MBRs, which correspond to objects appearing in an image. Figure 4 shows the extended relations between two intervals, $t$ and $u$. In the figure, $n 1$ and $n 2$ are $u . s t p-t . s t p$ and $u . e t p-t . e t p$, respectively.

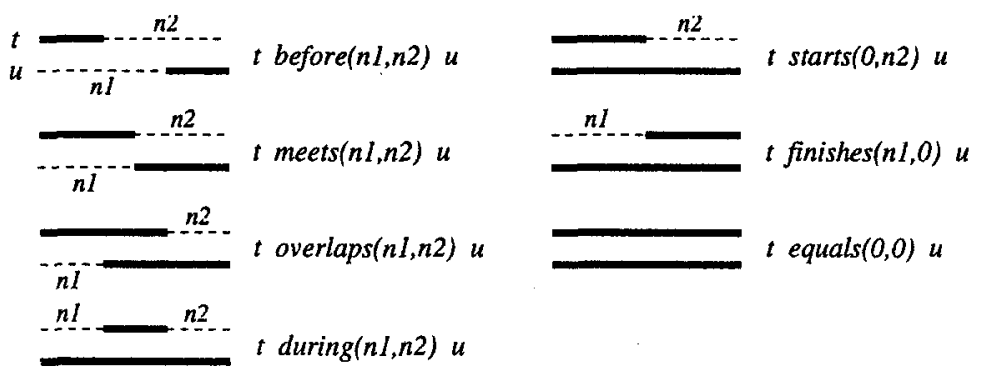

Fig. 4. Extended relations between two intervals

The extension allows us to give directional relations and distances between objects easily. Moreover, changes in an object can be described, with the values $n 1$ and $n 2$ used to express the exact degree of change. Note that this extension is natural and does not require anything of Allen's relations: the values $n 1$ and $n 2$ can be derived from the values of MBCs, which should already be stored in a database, and thus require no further expenditure of memory. If $t=\varepsilon$ and/or $u=\varepsilon$, the relation between them is not defined.

The relation between MBRs $O$ and $P$ is described as a combination of two relations, $R x\left(n 1_{x}, n 2_{x}\right)$ and $R y\left(n 1_{y}, n 2_{y}\right)$ (Fig. 5). The former represents the relation between the two intervals created by projecting the two objects on the $\mathrm{X}$-axis, and the latter that from projection on the $\mathrm{Y}$-axis. 


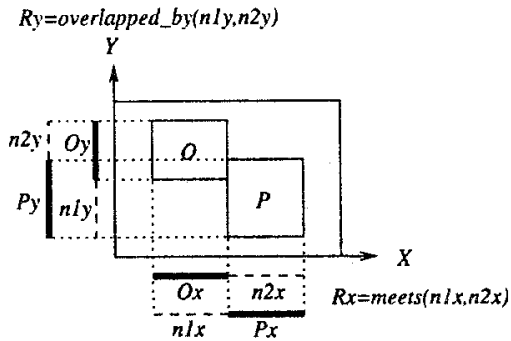

Fig. 5. An example of minimum bounding rectangle relations

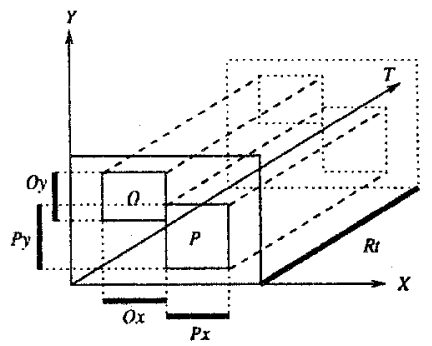

Fig. 6. An example of spatio-temporal cuboid relations

\section{Definition 8 Minimum bounding rectangle relations}

Given objects $O$ and $P$, let $(O x, O y ; O)$ and $(P x, P y ; P)$ be $M B R$ s of $O$ and $P$, respectively. Let $R x$ be the relation between $O x$ and $P x$, and $R y$ be that between $O y$ and $P y$. The MBR relation between the two objects is then defined as $(R x, R y ; O, P)$.

If $O x=O y=\varepsilon$ and/or $P x=P y=\varepsilon$, the relation between the two objects is not defined.

The following definition accounts for the time interval in which the MBR relation holds (Fig. 6).

\section{Definition 9 Spatio-temporal cuboid relations}

Given objects $O$ and $P$, let $(R x, R y ; O, P)$ be the $M B R$ relation between the $M B R s$ modeling the two objects. Let $R$ t be the time interval in which the relation holds. The spatio-temporal cuboid relation between the MBRs is then defined as $(R x, R y, R t ; O, P)$.

A spatio-temporal cuboid relation $(R x, R y, R t ; O, P)$ indicates that the MBR relation holds in the time interval $R t$. Therefore, we model changes in spatial relations between two objects using a collection of real time intervals (Fig. 7).

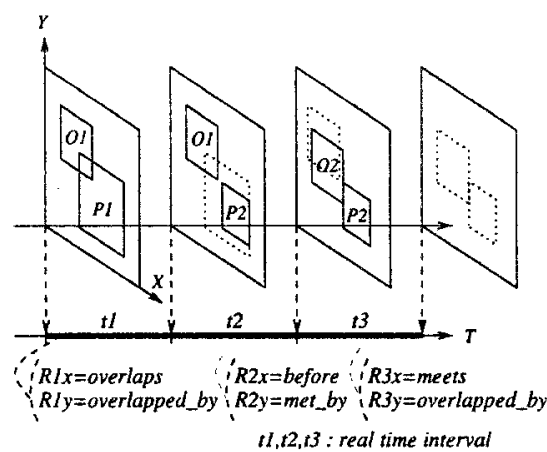

Fig. 7. Modeling changes in MBR relations between objects

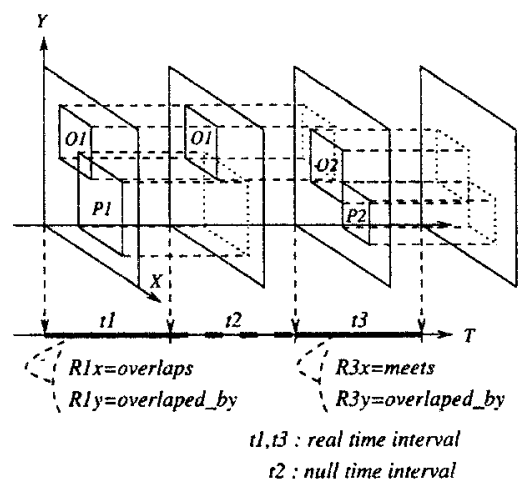

Fig. 8. A composite spatio-temporal cuboid relation

Lastly, let us define a collection of spatio-temporal cuboid relations, or a composite spatio-temporal cuboid relation, for modeling dynamic changes (Fig. 8). 
Definition 10 Composite spatio-temporal cuboid relations

Given objects $O$ and $P$, let $R_{i}=\left(R x_{i}, R y_{i}, R t_{i} ; O, P\right)(1 \leq i \leq n)$ be the spatio-temporal cuboid relation between them. The collection $\left\{R_{1}, R_{2}, \ldots, R_{n}\right\}$ is then defined as the composite spatio-temporal cuboid relation between them.

\subsection{Topological Relations}

There are eight kinds of topological relations between MBRs [12], as depicted in Fig. 9. In this study, we represent MBR relations with combinations of two relations between two intervals, as described earlier. Table 1 gives the topological relations based on the representation ${ }^{3}$. For example, given two objects, let the relation between their corresponding MBRs on the X-axis be contains and on the $\mathrm{Y}$-axis be starts; then the topological relation is Overlaps.

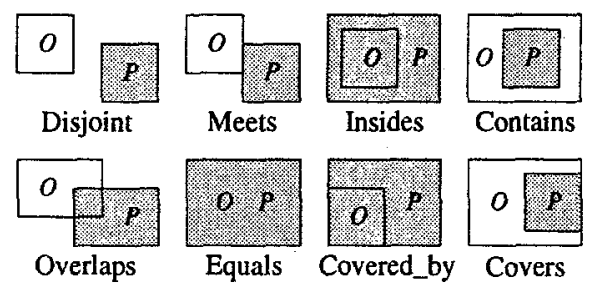

Fig. 9. Topological relations between two MBRs

Table 1. Topological relations between two MBRs

\begin{tabular}{|c|c|c|c|c|c|c|c|c|}
\hline & $\mathrm{b}|\mathrm{m}| \mathrm{o} \mid \mathrm{fb}$ & & & \begin{tabular}{l|l}
$\mathrm{e}$ & $\mathrm{st}$
\end{tabular} & d & & $o b \mid m$ & $\mathrm{ab}|\mathrm{a}|$ \\
\hline before & \begin{tabular}{|l|l|l|l}
$\mathrm{D}$ & $\mathrm{D}$ & $\mathrm{D}$ & $\mathrm{D}$ \\
\end{tabular} & D & $\overline{\mathrm{D}}$ & \begin{tabular}{l|l}
$D$ & $D$ \\
\end{tabular} & $\overline{\mathrm{D}}$ & \begin{tabular}{l|l}
$\mathrm{D}$ &
\end{tabular} & \begin{tabular}{l|l}
$\mathrm{D}$ & $\mathrm{I}$ \\
\end{tabular} & \begin{tabular}{l|l}
$\mathrm{D}$ & $\mathrm{D}$ \\
\end{tabular} \\
\hline meets & \begin{tabular}{|l|l|l|l|l|}
$\mathrm{D}$ & $\mathrm{M}$ & $\mathrm{M}$ \\
\end{tabular} & $\bar{M}$ & $\overline{\mathrm{M}}$ & \begin{tabular}{|l|l|}
$M$ & $M$ \\
\end{tabular} & $\bar{M}$ & & $\bar{M}$ & $\bar{M}|\mathrm{D}|$ \\
\hline overlaps & \begin{tabular}{|l|l|l|l|l|l|}
$\mathrm{D}$ & $\mathrm{M}$ & $\mathrm{O}$ \\
\end{tabular} & O & 0 & \begin{tabular}{|l|l}
0 & 0 \\
\end{tabular} & $\mathrm{O}$ & O & $\mathrm{O}$ & \begin{tabular}{l|l|}
$\mathrm{M} \mid \mathrm{D}$ \\
.
\end{tabular} \\
\hline finished_by & \begin{tabular}{|l|l|l|l|}
$\mathrm{M}$ & $\mathrm{CV}$ \\
\end{tabular} & $\mathrm{Cv}$ & $\overline{0}$ & $\mathrm{Cv} \mid \mathrm{C}$ & 0 & 0 & $\mathrm{O}$ & $\mathrm{M} \mid \mathrm{D}$ \\
\hline contains & 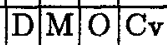 & $\mathrm{Ct}$ & Or & $\mathrm{Cv} \mid \mathrm{C}$ & 0 & $\mathrm{O}$ & \begin{tabular}{l|l}
0 & 1 \\
\end{tabular} & \begin{tabular}{l|l}
$\mathrm{M}$ & $\bar{D}$
\end{tabular} \\
\hline starts & \begin{tabular}{|l|l|l|l|}
$\mathrm{D}$ & $\mathrm{M}$ & $\mathrm{O}$ \\
\end{tabular} & 0 & $\overline{\mathrm{Cb}}$ & \begin{tabular}{|l|l}
$\mathrm{Cb}$ & $\mathrm{O}$ \\
\end{tabular} & 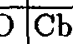 & $\mathrm{Cb}$ & \begin{tabular}{l|l}
0 & 1
\end{tabular} & $\mathrm{M} \mid \mathrm{D}$ \\
\hline equals & 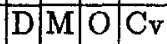 & $\mathrm{Cv}$ & $\mathrm{Cb}$ & \begin{tabular}{|l|l|}
$E$ & $C$ \\
\end{tabular} & $\begin{array}{ll}\mathrm{V} & \mathrm{Cb}\end{array}$ & $\mathrm{Cb}$ & $\mathrm{O}$ & $\overline{\mathrm{M}} \mid \mathrm{D}$ \\
\hline started_by & \begin{tabular}{|l|l|l|l|}
$\mathrm{D}$ & $\mathrm{Cr}$ \\
\end{tabular} & $\mathrm{Cv}$ & OR & \begin{tabular}{l|l}
$\mathrm{Cv}$ & $\mathrm{C}$
\end{tabular} & $\begin{array}{ll}\mathrm{V} & \mathrm{O}\end{array}$ & $\mathrm{O}$ & $\mathrm{O}$ & \begin{tabular}{l|l}
$M$ & $D$
\end{tabular} \\
\hline $\mathrm{ig}$ & \begin{tabular}{|l|l|l|l|l|}
$\mathrm{D}$ & $\mathrm{M}$ & $\mathrm{O}$ & $\mathrm{O}$ \\
\end{tabular} & 0 & $\mathrm{Cb}$ & \begin{tabular}{l|l}
$\mathrm{Cb}$ & $\mathrm{C}$ \\
\end{tabular} & \begin{tabular}{l|l|}
0 & 1 \\
\end{tabular} & $\mathrm{Cb}$ & $\begin{array}{ll}0 & 1\end{array}$ & \\
\hline finishes & 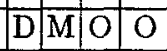 & $\mathrm{O}$ & $\mathrm{Cb}$ & \begin{tabular}{|l|l|}
$\mathrm{Cb}$ & $\mathrm{C}$ \\
\end{tabular} & $\mathrm{Cb}$ & $\mathrm{Cb}$ & 01 & \begin{tabular}{l|l}
$\mathrm{M}$ & $\mathrm{D}$ \\
\end{tabular} \\
\hline verlapped_by & $\mathrm{D} / \mathrm{M} O \mathrm{O}$ & 0 & 0 & \begin{tabular}{l|l}
$\mathrm{O}$ & $\mathrm{C}$ \\
\end{tabular} & $\begin{array}{ll}5 & 0\end{array}$ & 0 & $\bar{O}$ & \begin{tabular}{l|l}
$\mathrm{M} \mid \mathrm{D}$ \\
\end{tabular} \\
\hline met_by & \begin{tabular}{|l|l|l|l|l}
$D$ & $M$ & $M$
\end{tabular} & $\bar{M}$ & $\mathrm{M}$ & \begin{tabular}{|l|l|}
$M$ & $M$ \\
\end{tabular} & \begin{tabular}{l|l}
$\mathrm{M}$ & $\mathrm{M}$ \\
\end{tabular} & & $\overline{\mathbf{M}}$ & \begin{tabular}{l|l}
$\bar{M}$ & $\mathrm{D}$ \\
\end{tabular} \\
\hline after & \begin{tabular}{|l|l|l|l|l|}
$\mathrm{D}$ & $\mathrm{D}$ & $\mathrm{D}$ & $\mathrm{D}$ \\
\end{tabular} & $\mathrm{D}$ & D & \begin{tabular}{|l|l|}
$D$ & $\bar{D}$ \\
\end{tabular} & \begin{tabular}{l|l}
$D$ & $D$
\end{tabular} & & \begin{tabular}{l|l}
$\mathrm{D}$ & 1 \\
\end{tabular} & \begin{tabular}{l|l}
$D$ & $D$
\end{tabular} \\
\hline \begin{tabular}{|l|l|}
$D$ & Disjoint
\end{tabular} & & & & & & & & \\
\hline $\mathrm{M} \mid \mathrm{N}$ & & & & & & & & \\
\hline
\end{tabular}

The following lemmas are derived from Table 1.

\section{Lemma}

1. $(R x, R y ; O, P)=(R y, R x ; O, P)$

2. $(R x, R y ; O, P)=T R \Rightarrow\left(R x^{-1}, R y^{-1} ; O, P\right)=T R^{-1}$

${ }^{3}$ The same table can also be found in [14]. 
where

$$
T R^{-1}= \begin{cases}T R & (T R=\text { Disjoint, Meets, Overlaps, and Equals }) \\ \text { Contains } & (T R=\text { Insides }) \\ \text { Covered_by } & (T R=\text { Covers }) \\ \text { Insides } & (T R=\text { Contains }) \\ \text { Covers } & (T R=\text { Covered_by })\end{cases}
$$

\subsection{Directional Relations}

This subsection defines directional relations based on the relations shown in Fig. 4. There are two directional relations between two MBRs corresponding to two objects: one is on the $\mathrm{X}$-axis and the other on the $\mathrm{Y}$-axis.

\section{Definition 11 Directional relations}

Given objects $O$ and $P$, let $O i=(O i . s p, O i . e p)$ and $P i=(P i . s p, P i . e p)$ $(i=x$ or $y)$ be intervals created by projecting objects onto the axis of coordinates. Let us define that, from the $P$ 's point of view, object $O$ is in a negative direction from the axis if the following holds:

$$
\text { Oi.ep } \leq \frac{(P i . s p+P i . e p)}{2} \text {. }
$$

Also, let us define that object $O$ is in a positive direction from the axis from the $P$ 's point of view if the following holds:

$$
\frac{(\text { Pi.sp }+ \text { Pi.ep })}{2} \leq \text { Oi.sp . }
$$

Table 2 shows directional relations between two MBRs on the X-axis. In the table, $O<P$ means that object $O$ is in a negative direction from $P^{\prime}$ s point of view, and $O>P$ represents that $O$ is in a positive direction. On the other hand, $O=P$ indicates that the objects are in approximately the same place according to the definition of directional relations. The operation duration $(O x)$ returns the length of interval $O x$.

In this paper, when the directional relation $O<P$ on the $\mathrm{X}$ - and $\mathrm{Y}$-axes, we refer to this as $\operatorname{Left}(O, P)$ and $\operatorname{Below}(O, P)$, respectively, and when $O>P$ on the $\mathrm{X}$ - and $\mathrm{Y}$-axes, we refer to this as $\operatorname{Right}(O, P)$ and $A b o v e(O, P)$, respectively.

Note that the directional relations can be obtained from information regarding object $O$ and spatial relations between $O$ and $P$; thus, information regarding $P$ is not needed for deriving the directional relation of object $O$ from the viewpoint of object $P$.

Note also that the directional relation between objects can still be defined in cases where the topological relation between objects is not Disjoint or Meets. For example, let us consider the case illustrated in Fig. 10, where the relation between objects $P$ and $O$ is Insides, and where the following conditions hold:

$$
\begin{aligned}
\text { duration } & (O x)+\left|n 1_{x}\right| \leq n 2_{x} \\
\text { duration }(O y)+\left|n 1_{y}\right| & \leq n 2_{y}
\end{aligned}
$$

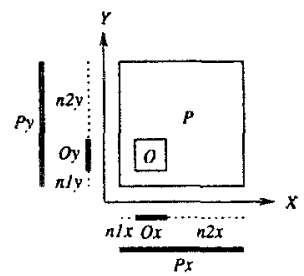

Fig. 10. An example of directional relations

In this case, object $O$ can be known to lie in a left-below direction from the viewpoint of object $P$. 
Table 2. Directional relations between two MBRs on the $\mathrm{X}$-axis

\begin{tabular}{l|l}
\hline before & $O<P$ \\
\hline meets & $O<P$ \\
\hline overlaps & $O<P$ if duration $(O x)-n 1_{x} \leq n 2_{x}$ \\
\hline during & $O<P$ if duration $(O x)+\left|n 1_{x}\right| \leq n 2_{x}$ \\
& $P<O$ if duration $(O x)+n 2_{x} \leq\left|n 1_{x}\right|$ \\
\hline starts & $O<P$ if duration $(O x) \leq n 2_{x}$ \\
\hline finishes & $P<O$ if duration $(O x) \leq\left|n 1_{x}\right|$ \\
\hline equals & $O=P$ \\
\hline finished_by & $O=P$ \\
\hline started_by & $O=P$ \\
\hline contains & $O=P$ \\
\hline overlapped_by & $P<O$ if duration $(O x)-\left|n 2_{x}\right| \leq\left|n 1_{x}\right|$ \\
\hline met_by & $P<O$ \\
\hline after & $P<O$ \\
\hline
\end{tabular}

\subsection{Distance between Objects}

The distance between two objects can also be defined with the MBR relations. This paper defines two kinds of distance.

\section{Definition 12 Distance between the centers of gravity 'Dist'}

Given two objects $O=(O x, O y ; O)$ and $P=(P x, P y ; P)$, let the $M B R$ relation between them be $\left(R x\left(n 1_{x}, n 2_{x}\right), R y\left(n 1_{y}, n 2_{y}\right) ; O, P\right)$. The distance between the centers of gravity $\operatorname{Dist}(O, P)$ is then defined as follows.

$$
\operatorname{Dist}(O, P)=\sqrt{X d i s t^{2}+Y d i s t^{2}}=\frac{1}{2} \sqrt{\left(n 1_{x}+n 2_{x}\right)^{2}+\left(n 1_{y}+n 2_{y}\right)^{2}}
$$

where

$$
\begin{aligned}
& \text { Idist }=\frac{(P i . s p+P i . e p)}{2}-\frac{(\text { Oi.sp }+ \text { Oi.ep })}{2}=\frac{(P i . s p-O i . s p)}{2}+\frac{(\text { Pi.ep }- \text { Oi.ep })}{2} \\
&=\frac{\left(n 1_{i}+n 2_{i}\right)}{2} \\
&((I, i)=(X, x) \text { or }(Y, y))
\end{aligned}
$$

Figure 11 depicts an example of the distance.

\section{Definition 13 Shortest distance between two objects 'SDist'}

Given two objects $O=(O x, O y ; O)$ and $P=(P x, P y ; P)$, let us assume that the topological relation between them is Disjoint. Let the MBR relation between them be $\left(R x\left(n 1_{x}, n 2_{x}\right), R y\left(n 1_{y}, n 2_{y}\right) ; O, P\right)$. Let us define Idist $(I=X$ or $Y)$ as follows.

$$
\text { Idist }= \begin{cases}n 1_{i}-\operatorname{duration}(O i) & R i=\text { before } \\ \left|n 2_{i}\right|-\operatorname{duration}(O i) & R i=\text { after } \\ 0 & \text { otherwise }\end{cases}
$$




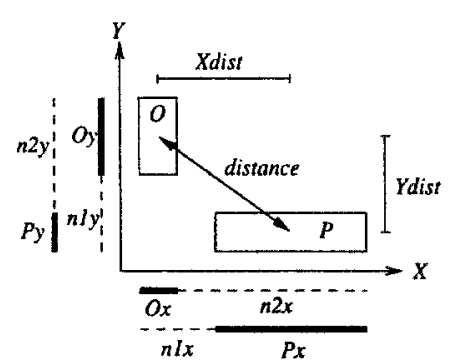

Fig. 11. An example of the distance between the centers of gravity

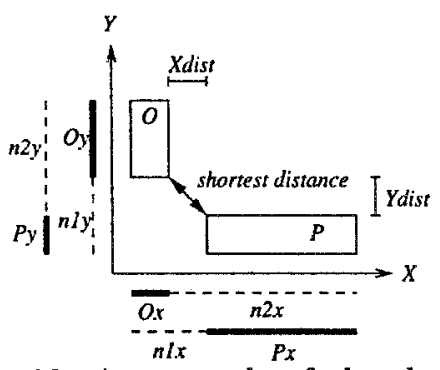

Fig. 12. An example of the shortest distance between two objects

where $i=x$ or $y$. Then, the shortest distance between the two objects SDist $(O, P)$. is defined as

$$
S \operatorname{Dist}(O, P)=\sqrt{X \operatorname{dist}^{2}+Y \operatorname{dist}^{2}}
$$

If the topological relation between two objects is Meets, then the shortest distance is defined as 0 . In the other cases, the shortest distance is not defined. and $P$.

Figure 12 illustrates an example of the shortest distance between objects $O$

\section{Representation of Image Sequences and Queries}

This section gives an example of representing an image sequence, and discusses the processing of queries.

\subsection{Representing an Image Sequence}

The following are the representations of objects $A, B$, and $C$ shown in Fig. 13 with composite spatio-temporal cuboids:

$$
\begin{aligned}
A= & \left\{\left((2,4),(9,11),\left(T_{1}, T_{2}-1\right) ; A\right),\left((2,6),(6,11),\left(T_{2}, T_{3}-1\right) ; A\right)\right. \\
& \left.\left((2,6),(4,8),\left(T_{3}, T_{5}-1\right) ; A\right)\right\} \\
B= & \left\{\left((6,8),(3,5),\left(T_{1}, T_{2}-1\right) ; B\right),\left(\varepsilon, \varepsilon,\left(T_{2}, T_{3}-1\right) ; B\right)\right. \\
& \left.\left((8,10),(4,6),\left(T_{3}, T_{4}-1\right) ; B\right),\left((7,9),(4,6),\left(T_{4}, T_{5}-1\right) ; B\right)\right\} \\
C= & \left\{\left((10,11),(8,9),\left(T_{1}, T_{5}-1\right) ; C\right)\right\}
\end{aligned}
$$

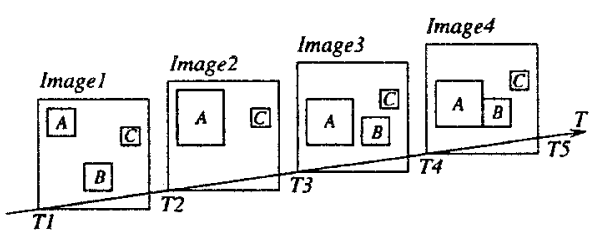

Fig. 13. An example of image sequences 
Using this representation, we can derive the composite spatio-temporal relations between any two of the three objects. We describe the composite spatiotemporal relation of $B$ from $A$ 's viewpoint as $A: B$, and derive it from the representation as follows.

$$
\begin{aligned}
A: B=\{ & \left\{\text { before }(4,4), \text { after }(-6,-6),\left(T_{1}, T_{2}-1\right) ; A, B\right), \\
& \left(\text { before }(6,4), \text { started_by }(0,-2),\left(T_{3}, T_{4}-1\right) ; A, B\right), \\
& \left.\left(\text { meets }(5,3), \text { started_by }(0,-2),\left(T_{4}, T_{5}-1\right) ; A, B\right)\right\}
\end{aligned}
$$

Also, the composite spatio-temporal relation can be used to derive the topological and directional relations, as well as the distances between objects. For example, the topological relation between $A$ and $B$ (referred to as $T R(A, B)$ ) can be obtained from Table 1 as follows.

$$
T R(A, B)=\left\{\begin{array}{l}
\text { Disjoint }\left\{\left(T_{1}, T_{2}-1\right),\left(T_{3}, T_{4}-1\right)\right\} \\
\text { Meets } \quad\left\{\left(T_{4}, T_{5}-1\right)\right\}
\end{array}\right.
$$

And the directional relation of $A$ from $B$ 's viewpoint (referred to as $D R(A, B)$ ) can be obtained from Table 2 as follows.

$$
D R(A, B)= \begin{cases}\text { LeftAbove } & \left\{\left(T_{1}, T_{2}-1\right)\right\} \\ \text { Left } & \left\{\left(T_{3}, T_{5}-1\right)\right\}\end{cases}
$$

Finally, the distances between $A$ and $B$ can also be calculated.

$$
\operatorname{Dist}(A, B)=\left\{\begin{array}{ll}
2 \sqrt{13} & \left\{\left(T_{1}, T_{2}-1\right)\right\} \\
\sqrt{26} & \left\{\left(T_{3}, T_{4}-1\right)\right\} \\
\sqrt{17} & \left\{\left(T_{4}, T_{5}-1\right)\right\}
\end{array} \quad \operatorname{SDist}(A, B)= \begin{cases}\sqrt{10} & \left\{\left(T_{1}, T_{2}-1\right)\right\} \\
1 & \left\{\left(T_{3}, T_{4}-1\right)\right\} \\
0 & \left\{\left(T_{4}, T_{5}-1\right)\right\}\end{cases}\right.
$$

\subsection{Spatial Queries}

We will examine spatial queries with regard to four types of information: MBR relations, topological relations, directional relations, and distances between objects. More general spatial queries can be written by combining queries of the four types.

1. Minimum bounding rectangle relations $\left(R x\left(n 1_{x}, n 2_{x}\right), R y\left(n 1_{y}, n 2_{y}\right) ; O, P\right)$ $\left(n i_{j}\right.$ ( $i=1$ or $2, j=x$ or $y$ ) can be omitted)

Ex. Which image has the relation (before(), after();A,B) ?

$$
\text { (before }(), \operatorname{after}() ; A, B) \Rightarrow\left\{\left(T_{1}, T_{2}-1\right)\right\} \Rightarrow \text { Image } 1
$$

2. Topological relations $T R(O, P)$

Ex. Which image has the relation $\operatorname{Meets}(A, B)$ ?

$$
\operatorname{Meets}(A, B) \Rightarrow\left\{\left(T_{4}, T_{5}-1\right)\right\} \Rightarrow \operatorname{Image} 4
$$

3. Directional relations $D R(O, P)$

Ex. Which image has the relation $\operatorname{Left}(A, B)$ ?

$$
\operatorname{Left}(A, B) \Rightarrow\left\{\left(T_{1}, T_{2}-1\right),\left(T_{3}, T_{5}-1\right)\right\} \Rightarrow \text { Image } 1, \text { Image } 3, \text { Image } 4
$$

4. Distances between objects $\operatorname{Dist}(O, P)$ and $S \operatorname{Dist}(O, P)$

Ex. Which image has objects $A$ and $B$ where the shortest distance between them is equal to or less than 2 ?

$$
S \operatorname{Dist}(A, B) \leq 2 \Rightarrow\left\{\left(T_{3}, T_{5}-1\right)\right\} \Rightarrow \text { Image } 3 \text {, Image } 4
$$




\subsection{Temporal Queries}

Queries about an Object In this subsubsection, we discuss queries about an object; that is, about a composite spatio-temporal cuboid. Using such queries, we can express queries about dynamic changes of an object. Here we discuss the following five kinds of changes: appearance, disappearance, expansion, contraction, and movement.

- Appearance

Given a composite spatio-temporal cuboid, if there is relation meets between a null-time interval and a real-time interval, then the start time point of the real-time interval defines the appearance of the object.

Ex. When does object $B$ appear? $\Rightarrow T_{3}$

- Disappearance

Given a composite spatio-temporal cuboid, if there is relation meets between a real-time interval and a null-time interval, then the start time point of the null-time interval represents the disappearance of the object.

Ex. When does object $B$ disappear? $\Rightarrow T_{2}$

In the following, we refer to object $A$ as $A 1=(A 1 x, A 1 y ; A 1)$ before changing, and as $A 2=(A 2 x, A 2 y ; A 2)$ after changing. To introduce the remaining changes, we project the time interval of $A 2$ to the time interval of $A 1$, and let $\left(R x\left(n 1_{x}, n 2_{x}\right), R y\left(n 1_{y}, n 2_{y}\right) ; A 1, A 2\right)$ be the MBR relation between $A 1$ and $A 2$ after the projection.

- Expansion

Let us define that object $A$ expands if the following holds:

$$
A 1 x\left(n 2_{y}-n 1_{y}\right)+\left(n 2_{x}-n 1_{x}\right)\left(A 1 y+n 2_{y}-n 1_{y}\right)>0 .
$$

Ex. When does object $A$ expand? $\Rightarrow T_{2}$

- Contraction

Let us define that object $A$ contracts if the following holds:

$$
A 1 x\left(n 2_{y}-n 1_{y}\right)+\left(n 2_{x}-n 1_{x}\right)\left(A 1 y+n 2_{y}-n 1_{y}\right)<0 \text {. }
$$

Ex. When does object $A$ contract? $\Rightarrow T_{3}$

- Movement

Let us define that object $A$ moves if the following holds:

$$
\left(n 1_{x}+n 2_{x}\right)^{2}>0 \vee\left(n 1_{y}+n 2_{y}\right)^{2}>0 .
$$

Ex. When does object $A$ move? $\Rightarrow T_{4}$

Queries about Spatio-Temporal Relations between Objects This subsubsection discusses queries about dynamic changes of spatial relations between objects. This kind of query requests a collection of time intervals that satisfy predicates. It can thus be expressed using temporal relations between collections of time intervals. Here, let us define the temporal relations between collections of time intervals.

Definition 14 Temporal relations between collections of time intervals

Let $C_{1}=\left\{t_{1}, \ldots, t_{m} \mid t_{i}(1 \leq i \leq m)\right.$ is a time interval $\}$ and $C_{2}=\left\{u_{1}, \ldots, u_{n} \mid\right.$ $u_{j}(1 \leq j \leq n)$ is a time interval $\}$ be collections of time intervals. We define that $R_{T E M P}\left(C_{1}, C_{2}\right)$, where $R_{T E M P}$ is a relation between intervals, is true if the following holds:

$$
\exists t_{i} \exists u_{j} \quad t_{i} R_{T E M P} u_{j} .
$$


Ex. Is it true that object $A$ is first apart from object $B$ and then touches it?

$$
\begin{aligned}
& \operatorname{after}(\operatorname{Disjoint}(A, B), \text { Meets }(A, B)) \\
& \Rightarrow \operatorname{after}\left(\left\{\left(T_{1}, T_{2}-1\right),\left(T_{3}, T_{4}-1\right)\right\},\left\{\left(T_{4}, T_{5}-1\right)\right\}\right) \\
& \Rightarrow \operatorname{true}
\end{aligned}
$$

Ex. Is it true that object $A$ is first to the right of object $B$ and next to the left of it?

$$
\begin{aligned}
& \text { after }(\operatorname{Right}(A, B), \operatorname{Left}(A, B)) \\
& \Rightarrow \operatorname{after}\left(\{\emptyset\},\left\{\left(T_{1}, T_{2}-1\right),\left(T_{3}, T_{5}-1\right)\right\}\right) \\
& \Rightarrow \text { false }
\end{aligned}
$$

\section{Related Work}

Nabil et al. [12] defined 2D Projection Interval Relationships for representing directional and topological relationships between two objects. They adapted Allen's temporal intervals [1] and 2D-strings to produce the unified representation. In their study, explicit directional relationships were not taken into account, because their representation treats these relationships implicitly. As a result, if there is an overlap relationship between objects, their model cannot directly express it, and cannot write queries to derive it. Moreover, they did not integrate temporal concepts into their work.

Del Bimbo et al. [5] proposed a language, Spatio-temporal Logic, for the symbolic representation of the contents of image sequences. In this logic, objects are represented as MBRs, and spatial relationships between two objects are defined as combinations of Allen's interval relations along the $x$ - and $y$-axes. However, they treated only orders for temporal representation and not including times of occurrence, and directly expressed neither time intervals in which an event occurs nor those in which an event suspends. On the other hand, changes of spatial relations can be calculated by applying queries to the simple recorded information in our study, and any kinds of spatio-temporal queries can also be expressed.

Claramunt and Thériault [3] introduced several kinds of changes of each object appearing in images, and proposed recording them along with the times of their occurrence. However, their model cannot express the exact degree of changes. In contrast, we proposed a method for enhancing the representation of changes, thereby allowing for greater verisimilitude.

The study by Day et al. [4] is probably the most similar to our own. These authors proposed a graphical data model for specifying spatio-temporal semantics of video data. In the model, MBRs are used not only to model objects, but to derive the relative spatial relationships between them. One point of difference between their study and the present one is that, again, they did not intend to express the exact degree of change.

\section{Conclusions and Future Research}

In this paper we have proposed an interval-based method of representing spatiotemporal concepts. The discussion has revealed mainly the following:

- Since objects are modeled after MBRs, we can easily represent positions and sizes of objects, and state the spatial relations between them.

- The time-interval based conceptual model allows us to express dynamic changes of MBRs used to model objects and of the spatial relations between them. 
- Extended relations between two intervals make it possible to treat topological and directional relations and the distances between objects.

We have also illustrated how spatio-temporal queries are performed in the proposed method.

There still exist several kinds of dynamic changes that the proposed method cannot express, e.g., split-ups and attachment of objects. We will continue its development in order to overcome these problems. Moreover, we intend to design and implement an appropriate query language for use with the new intervalbased technique.

\section{References}

1. J.F. Allen, "Maintaining Knowledge about Temporal Intervals," $C A C M, 26(11)$, pp.832-843, Nov. 1983.

2. T. Amagasa, M. Aritsugi, Y. Kanamori, and Y. Masunaga, "Interval-Based Modeling for Temporal Representation and Operations," Submitted for Pub., 1997.

3. C. Claramunt and M. Thériault, "Managing Time in GIS An Event-Oriented Approach," Proc. the Int. Workshop on Temporal Databases, pp.23-42, Sep. 1995.

4. Y.F. Day, S. Dağtaş, M. Iino, A. Khokhar, and A. Ghafoor, "Object-Oriented Conceptual Modeling of Video Data," Proc. 11th ICDE, pp.401-408, 1995.

5. A. Del Bimbo, E. Vicario, and D. Zingoni, "Symbolic Description and Visual Querying of Image Sequences Using Spatio-Temporal Logic," IEEE TKDE, 7(4), pp.609-621, 1995.

6. M.J. Egenhofer, "Spatial SQL: A Query and Presentation Language," IEEE TKDE, 6(1), pp.86-95, 1994.

7. N. Fukuda, T. Amagasa, Y. Kanamori, and Y. Masunaga, "Extended Data Model based on Temporal Intervals," Proc. Advanced Database System Symposium '94, pp.175-184, Dec. 1994 (in Japanese).

8. S.L. Hibino and E.A. Rundensteiner, "Interactive Visualizations for Exploration and Spatio-Temporal Analysis of Video Data," Position Paper for IJCAI'95 Workshop on Intelligent Multimedia Information Retrieval, Feb. 1995.

9. C.S. Jensen, et al., "A Consensus Glossary of Temporal Database Concepts," SIGMOD RECOR.D, 23(1), pp.52-63, Mar. 1994.

10. P. Ladkin, "Time Representation: A Taxonomy of Interval Relations," Proc. the National conference on Artificial Intelligence, pp.360-366, 1986.

11. M. Nabil, A.H.H. Ngu, and J. Shepherd, "Picture Simularity Retreival Using the 2D Projection Interval Representation," IEEE TKDE, 8(4), pp.533-539, Aug. 1996.

12. M. Nabil, J. Shephred, and A.H.H. Ngu, "2D-Projection Interval Relationships: A Symbolic Representation of Spatial Relationships," Proc. 4th International Symposium on Large Spatial Databases, pp.292-309, Aug. 1995.

13. D. Papadias and T. Sellis, "Qualitative Representation of Spatial Knowledge in Two-Dimensional Space," VLDB Journal, 3(4), pp.479-516, Oct. 1994.

14. D. Papadias, Y. Theodoridis, T. Sellis, and M.J.Egenhofer, "Topological Relations in the World of Minimum Bounding Rectangles: A Study with R-trees," Proc. SIGMOD Conf., pp.92-103, June 1995.

15. R.T. Snodgrass Ed., The TSQL2 Temporal Query Language, Kluwer Academic Publishers, 1995. 